\title{
EL TRABAJO INFANTIL Y SUS REPERCUSIONES EN EL DESARROLLO INTEGRAL DE LAS PERSONAS MENORES DE EDAD
}

\author{
Maria Ester Morales Ramirez*
}

Se reflexiona en cuanto al impacto que el Trabajo Infantil tiene en el Desarrollo Integral de los niños, niñas y adolescentes, específicamente, en el aspecto físico, emocional, social, moral y cognitivo. Esta actividad luego tiene repercusiones más graves especialmente cuando las personas menores de edad desertan del sistema educativo para dedicarse a ella. La reflexión señala el Trabajo Infantil como una de las principales causas de explotación y abuso de este grupo de población y cuestiona el rol de cada uno de nosotros en el manejo de esta situación.
The article considers the impact of child labour (Child work exploitation) in the adequate integral development of boys, girls and teenagers, specifically on aspects related to their physical, emotional, social, moral and cognitive growth. This type of activity shows serious consequences when youngsters abandon the educational system to dedicate their time to work.

This paper points out child labour as one of the main causes of exploitation and child abuse in this population group, and questions our role, as citizens, towards the management of this situation.

* Master en Psicología Industrial y Organizacional por la Universidad Latina de Costa Rica. Licenciada y Bachiller en Psicología por la Universidad de Costa Rica.

Labora como Académica y coordina el Programa "Apoyo Psicopedagógico a la Educación Formal y no Formal” en el Instituto de Estudios Interdisciplinarios de la Niñez y la Adolescencia del CIDE, Universidad Nacional. 
La Convención sobre los Derechos del Niño, aprobada por la Asamblea General de las Naciones Unidad, el 20 de noviembre de 1989 y ratificada por Costa Rica en 1990, establece como uno de sus principios la supervivencia y desarrollo, específicamente en su artículo 6 apunta que:

1. "Los Estados Partes reconocen que todo niño tiene el derecho intrínseco a la vida".

2. "Los Estados Partes garantizarán en la máxima medida posible la supervivencia y el desarrollo del niño" (p.3).

De igual manera, plantea como principio (que respalda al Artículo 32), lo siguiente "es obligación del Estado proteger al niño contra el desempeño de cualquier trabajo nocivo para su salud, educación o desarrollo; fijar edades mínimas de admisión al empleo y reglamentar las condiciones del mismo" (p.11).

Al respecto, el Código de la Niñez y la Adolescencia de Costa Rica (1998), refiere en su artículo 7, lo que corresponde al desarrollo integral de la persona menor de edad en el sentido que:

La obligación de procurar el desarrollo integral de la persona menor de edad les corresponde, en forma primordial a los padres o encargados, las instituciones integrantes del Sistema Nacional de Protección Integral de la Niñez y la Adolescencia, regúlado en el título IV de este Código, garantizarán el respeto por el interés superior de estas personas en toda decisión pública o privada. La Defensoría de los Habitantes de la República velará por el cumplimiento efectivo de estas obligaciones" (p.67) y en lo que corresponde a la Protección de los adolescentes trabajadores establece en el artículo 78 que El Estado reconocerá el derecho de las personas adolescentes mayores de quince años a trabajar con las restricciones que imponen este Código, los convenios internacionales y la ley. Este derecho podrá limitarse solamente cuando la actividad laboral imparte riesgo, peligro para el desarrollo, la salud física, mental y emocional o cuando perturbe la asistencia regular al centro educativo (p.89).

Como puede verse, en nuestro país existen leyes, convenios y otros, cuyo fin es proteger a las personas menores de edad, hacer viable el respeto por sus derechos y propiciar las condiciones necesarias para garantizar el desarrollo integral de estos.

Para ello la Organización Internacional del Trabajo (OIT, 1996), apunta que, desde principios de los años noventa, al problema del trabajo infantil se le ha prestado mayor atención. Esto debido a la concienciación en el sentido que: 
- La explotación de los niños en el trabajo podría ser cada vez más grave en diferentes partes del mundo, producto del deterioro de las condiciones económicas y de las serias repercusiones que esta situación tiene en el desarrollo social, especialmente en el empleo y la educación.

- Preocupa el hecho de que en algunos países se aprovechan del trabajo de los niños de edades y condiciones contrarias a lo establecido por la normativa. para tener ventaja en el campo del comercio internacional, en comparación con otros países y,

- Cada vez son más las luchas que se dan en relación con la defensa de los derechos del niño.

Agrega la OIT que, el trabajo infantil se da principalmente en países en desarrollo, aunque esto no significa que en países industrializados esta situación no se presente. Asimismo, apunta que la participación de los niños, niñas y adolescentes en la actividad económica, se da igualmente en las zonas rurales y urbanas del país y que el trabajo infantil no remunerado en empresas familiares, podría ser más frecuente en el medio rural que en el medio urbano, lugar éste donde es posible encontrar el mayor número de trabajadores asalariados.

Para la OIT se ha dado un cambio de actitud en relación con el trabajo infantil, ya que este anteriormente era percibido como un medio más que contribuía en el proceso de socialización del niño y por ende, le permitía un mejor desenvolvimiento en el futuro. No obstante, hoy en día, las familias ven el trabajo infantil como la alternativa para obtener ingresos económicos complementarios.

Se señala que no se cuenta con muchos datos acerca de las condiciones en que los niños realizan el trabajo, así como los riesgos o abusos a que están expuestos. Esto, obviamente conlleva a que no se tenga absoluta claridad en cuanto a cuáles son los trabajos que están desempeñando y que podrían perjudicarles en su proceso de desarrollo integral.

Como motivo de gran preocupación se agrega que los niños empiezan a trabajar muy jóvenes y principalmente en el medio rural donde podrían iniciar su labor productiva a los cinco o seis años. Al parecer, la mayor cantidad de niños económicamente activos pertenecen al grupo de edad comprendido entre los 10 y los 14 años.

Otro elemento de preocupación para la OIT, es que la mayoría de las veces el trabajo se constituye en una actividad permanente para los niños, limitándoles de esta manera el acceso a la educación en condiciones satisfactorias. A diferencia de los países industrializados, donde los niños se insertan al trabajo esporádicamente, es decir, fines de semana o en períodos de vacaciones con el propósito de obtener dinero para sus gastos personales, en los países en desarrollo, las personas menores de edad deben trabajar para obtener los recursos que les permita vivir o sobrevivir diariamente. 
Aunado a lo anterior, está el hecho de que muchos niños, que son económicamente activos, van también a la escuela. Sin embargo, no todos lo hacen, por lo que el porcentaje de los que desertan o no van del todo a la escuela es alarmante. Aún cuando no se tienen cifras concretas, esto es motivo de análisis, especialmente, porque se desconoce si los niños abandonan su educación porque están obligados a trabajar, o si deben trabajar porque desertan de la escuela por otras razones.

Se hace referencia, además, al cansancio y agotamiento que experimentan los niños, producto de la cantidad de horas que les corresponde trabajar. Si bien estas deben ser consecuentes con la edad y el tipo de trabajo que desempeñan, de no tenerse control al respecto, se afectaría considerablemente la capacidad de aprendizaje de los niños.

\section{Causas del trabajo infantil}

La Organización Internacional del Trabajo (1996), señala entre otras las siguientes:

- La pobreza de las familias, esto hace que los niños tengan que trabajar a tiempo completo para poder obtener los ingresos necesarios que les permita vivir a ellos y a sus familias.

- El acceso a la educación puede ser muy difícil para algunas familias,.aún cuando la educación pública es gratuita, esta puede ser cara para algunas familias pobres que no poseen los medios para comprar los libros, los uniformes, los artículos escolares, el transporte y otros pagos que establece el Centro Educativo.

- Con frecuencia se dificulta el acceso de muchos niños a los Centros Educativos debido a lo aisladas que se encuentran las comunidades donde viven, por lo que trabajan en vez de estudiar. Aunado a esto, está la calidad de la educación que se brinda en algunas escuelas, por lo que algunos padres y niños se desmotivan ante el estudio, debido a que la información y formación que reciben no responde a sus intereses y necesidades y tampoco los prepara para su adecuado desempeño laboral en el futuro.

- Las familias pobres con frecuencia son más numerosas, tienen más hijos, lo que conlleva a que estos deban trabajar para aumentar los ingresos del hogar que les permita satisfacer sus necesidades y sobrevivir.

¿Qué repercusiones tiene esta situación en el desarrollo integral de las personas menores de edad? y más específicamente, ¿de qué manera les impacta el 
insertarse a temprana edad en el ámbito laboral. sean las condiciones del trabajo favorables o no?

En un intento de dar respuesta a las preguntas antes planteadas, es necesario tener claridad respecto a qué entendemos por desarrollo integral y de esta manera poder conocer, aunque sea en forma general, cómo en este podrían presentarse momentos críticos que dificulten el proceso, especialmente, si el niño, la niña y los y las adolescentes están ejecutando una actividad que no favorece el desarrollo esperado para su edad.

\section{¿Qué se entiende por desarrollo integral?}

Al respecto, Trejos (1998) cita a León, quien define el desarrollo humano como (...) "todos los procesos y cambios que se dan en el individuo a partir de su concepción y hasta su muerte y que progresivamente van conformando lo que es el ser humano en sus diversas etapas" (p.2).

Cabe mencionar que el desarrollo humano no puede concebirse al margen de la historia biológica, de la herencia, y del contexto físico y social en que la persona se desenvuelve, por esto y para fines del presente trabajo y con el propósito de conocer el efecto que en el desarrollo de las personas menores de edad tiene la inserción temprana en el campo laboral, es necesario tener claro el rol fundamental que en este aspecto tienen la familia, la escuela, la sociedad y los medios de comunicación, entre otros.

Es función de las instancias mencionadas, el proporcionar los recursos y condiciones necesarias para que los niños, las niñas y los y las adolescentes logren su óptimo desarrollo integral; es decir, el óptimo desarrollo en el área física, social, intelectual y emocional.

\section{¿Cómo incide el trabajo infantil en el desarrollo de las personas menores de edad?}

Para la OIT (1996), el trabajo infantil es en el mundo de hoy, un problema preocupante, ya que cada vez son más los niños afectados y por las consecuencias negativas que tienen las inadecuadas condiciones del trabajo sobre el desarrollo integral de estos y sobre el desarrollo económico y social de los países involucrados.

Al respecto agrega la OIT (1996) que:

(...) Durante la infancia, el mundo que nos rodea nos ofrece oportunidades importantes para aprender. Desarrollamos habilidades que nos 
permiten ser seres sociales para participar plenamente en la vida familiar y comunitaria. Esta etapa de la vida es crítica e influye en nuestro futuro. Los trabajadores infantiles se ven privados de este período valioso. Su trabajo traba las actividades normales de la infancia y entorpece su desarrollo físico, emocional y social (p.20).

Acerca de este tema, en el informe del Estado Mundial de la Infancia (1997) se hace referencia al hecho de que no es el trabajo por sí mismo lo que afecta negativamente el desarrollo de los niños, sino la naturaleza y condiciones en que lo realizan.

Se agrega que los niños desempeñan su trabajo y las tareas que de este se derivan de maneras muy diversas, lo que conlleva a que para algunos esta actividad sea positiva y beneficiosa, ya que promueve el óptimo desarrollo físico, mental, espiritual, moral y social de estos, sin afectar su proceso de aprendizaje, ni sus espacios para la recreación y el descanso.

No obstante lo anterior, para la mayoría de los niños que trabajan, este se constituye en una actividad nociva, abusiva y violatoria de sus derechos a ser cuidados y protegidos por sus padres, a recibir una educación básica obligatoria y gratuita, a tener acceso a los servicios del sistema de salud, a la seguridad social y a los medios que le permitirán descanso y recreación.

En virtud del planteamiento precitado, se hace necesario discriminar cuándo un trabajo puede beneficiar al niño, a la niña y a los (as) adolescentes y cuándo se constituye en abuso y explotación.

Al respecto, en el informe del Estado Mundial de la Infancia (1997), se menciona que el Fondo de las Naciones Unidas para la Infancia (UNICEF), estableció que el trabajo infanto-juvenil adquiere dimensiones de explotación y abuso cuando se presentan las siguientes condiciones:

- trabajo a tiempo completo a una edad demasiado temprana,

- horario laboral prolongado,

- trabajo que produce tensiones indebidas de carácter físico, social o psicológico,

- trabajo y vida en la calle en malas condiciones, remuneración inadecuada,

- trabajos que obstaculizan el acceso a la educación,

- trabajos que socavan la dignidad y autoestima de los niños tales como la esclavitud o el trabajo servil y la explotación sexual y

- trabajos que perjudican el pleno desarrollo social y psicológico (p.24). 
Agregan que el trabajo infanto-juvenil puede afectar negativamente el desarrollo de estos grupos de población en los siguientes aspectos:

1. Desarrollo físico. Los niños, niñas y adolescentes que laboran en condiciones de explotación "se ven expuestos a sufrir graves daños en su desarrollo y madurez", lo que conlleva al deterioro de las condiciones de la salud en general. la coordinación, la resistencia, la visión y la audición.

Explican que a nivel mundial existen datos que confirman estos daños, especialmente cuando trabajan en "lugares contaminados" o en condiciones de alto riesgo y de jornadas que no son las que corresponden a su edad, lo que conlleva a la aparición de problemas serios en la salud, que la mayoría de las veces no ceden ante una eventual medida o tratamiento médico.

En el Estado Mundial de la Infancia (1997), se hace referencia a que el daño físico es el más fácil de percibir por cuanto el traslado de pesadas cargas o el tener que adoptar posiciones incómodas por largos períodos de tiempo, podrían "originar discapacidades permanentes en el organismo infantil en pleno desarrollo" (p.25).

Se agrega que si los niños, niñas y adolescentes desempeñan tareas que requieren de gran esfuerzo físico por largo tiempo, se verá afectada la talla hasta en un $30 \%$ de su potencial biológico. Esto por cuanto gastan la energía que debería "durarles hasta la edad adulta".

En relación con esta misma área del desarrollo, la OIT (s.f.) apunta que:

Los niños trabajadores son mucho más vulnerables que los adultos porque sus cuerpos todavía están creciendo y no están formados completamente. Tienen una salud física deficiente porque el trabajo que ejercen los expone a los riesgos de las enfermedades y los accidentes. Los efectos pueden ser inmediatos, como por ejemplo, una quemadura o un corte, o pueden tener consecuencias que duran toda la vida, como sufrir alguna enfermedad respiratoria o contraer el SIDA (p.20).

En el Estado Mundial de la Infancia (1997), se indica que en Manila los niños con frecuencia dedican hasta seis horas de trabajo escarbando en inmensos Vertederos de basura y que si bien esto les aporta un ingreso mayor que el que obtiene un adulto trabajando en una fábrica, la búsqueda de basura es un trabajo degradante y peligroso para los niños. Se agrega que el contexto en que se realiza este trabajo es antihigiénico, peligroso, vejatorio y destructivo para su autoestima. Además, se ven expuestos a todo tipo de condiciones climatológicas que les provoca neumonía, gripe e insolación, a infecciones en la piel y a tétanos u otras enfermedades. Por otra parte el transporte de cargas muy pesadas 
para su edad, dificulta su crecimiento y les puede producir lesiones irreversibles en el desarrollo de la columna vertebral, en su desarrollo en general y el hecho de que ingieran sobras de comida les causa frecuentes trastornos digestivos e intoxicaciones.

Se menciona en este mismo informe que otros niños se dedican a labores propias de la agricultura -tales como recoger café, caña, té y tabaco, entre otras, las que son igualmente peligrosas clescle el punto de vista de la salud y la seguridad, ya que los niños recogen las plantas todavía cubiertas de pesticidas o las riegan ellos mismos con este producto, lo que les provoca problemas dermatológicos, oculares, respiratorios o neurológicos. Además, están expuestos a picaduras cle serpientes e insectos venenosos, a herirse con los utensilios que utilizan y debido a lo húmedo del medio en que trabajan, es frecuente la aparición de infecciones respiratorias crónicas y neumonía.

2.Desarrollo emocional. Abarca la autoestima, la afectividad familiar y los sentimientos de aceptación y amor.

En el Estado Mundial de la Infancia (1997), se apunta que la mayoría de los niños, niñas y jóvenes que trabajan proceden de hogares en condiciones de pobreza y vulnerabilidad, por lo que los padres, respondiendo a patrones culturales, los insertan a temprana edad en labores que podrían conllevar a la explolación y al abuso, con el fin de que el clinero que devengan se constituya en un aporte más al ingreso familiar. En estos casos, la clinámica que se desarrolla al interior de las familias, podría inciclir negativamente en el clesarrollo de los vínculos afectivos entre sus miembros y por consiguiente en el fortalecimiento de su auto concepto, de su autoestima, de su autonomía, independencia y principalmente en tener la certeza de no ser queridos, aceptados y valorados, razón por la que no merecen ser protegidos, sobre todo cuandlo la familia les exige demasiado, clemandáncloles en ocasiones largas jornadas de trabajo que les imposibilitan tener el derecho a satisfacer sus propias necesidacles.

Lo anteriormente citado es reforzado por la OIT (s.f.) en el sentido que:

Los niños trabajadores frecuentemente laboran en actividades que son explotadoras, peligrosas, degradantes y cle aislamiento. Muy a menu(lo son maltralados, abusados y abandonados por sus patrones. Como consecuencia de esto, los niños podrían tener dificultades para vincularse con otras personas o tener resentimientos bacia ellas. Tienen problemas para interactuar y' cooperar con otros y para clesarrollar un sentido real cle identidad. A menudo les falta confianza en sí mismos y experimentan sentimientos cle baja auto-estima (p.20). 
Así mismo, la OIT refiere que las niñas que se dedican a trabajos domésticos, están privadas de afecto y del contacto con sus familiares y amigos. Además, en su mayoría, están expuestas a malos tratos físicos, psicológicos y a abusos sexuales y podrían presentar síntomas como retraimiento, regresión. envejecimiento prematuro y depresión, entre otros.

El que los niños, las niñas y adolescentes se enfrenten con frecuencia a estas experiencias, los va haciendo psicológicamente vulnerables, con una alta probabilidad de que caigan en un círculo de abuso, explotación, rechazo y opresión, del cual se les podría dificultar el poder salirse.

No ocurre lo mismo cuando las familias asignan tareas para ser ejecutadas por los niños, niñas y jóvenes, al interior de los hogares, las que podrían considerarse beneficiosas para el desarrollo por cuanto les permite adquirir experiencia, confianza en que pueden hacerlo y reconocimiento por parte de las familias. siempre que estas no los coloquen en condiciones de vulnerabilidad, de desprotección y de explotación que atenten contra sus derechos.

3. Desarrollo social y moral. El desarrollo en esta área puede verse afectada negativamente, en especial cuando los niños, las niñas y adolescentes realizan tareas en condiciones de desventaja que limitan su proceso de socialización. Según el Estado Mundial de la Infancia (1997), el no poder permanecer por largo tiempo con su familia ni con sus pares, conlleva a que no se desarrolle como corresponde el sentido de pertenencia e identidad grupal, una actitud de cooperación y colaboración para con los otros y la incorporación de límites y normas que les permitan tener claridad acerca de qué pueden o no hacer y qué es bueno y qué no lo es.

$\mathrm{Al}$ respecto la OIT (s.f.) apunta que:

Los niños que laboran no tienen la oportunidad de participar en actividades que son vitales para su formación, como por ejemplo, jugar, ir a la escuela y socializar con otros niños. No adquieren el nivel básico de educación que se necesita para enfrentar la vida. Tampoco tienen la oportunidad de interactuar con otras personas, ni participar activamente, ni gozar de la vida. Estas actividades son abandonadas a favor del trabajo y, consecuentemente, los niños se ven presionados a llevar una vida adulta sin que estén listos para ello, hacen un trabajo que requiere el nivel de madurez de un adulto (p. 20).

4. El desarrollo cognitivo o intelectual. Este según el Estado Mundial de la Infancia (1997), abarca "la alfabetización, el cálculo numérico y la adquisición de conocimientos necesarios para la vida ordinaria” (p.28). 
La UNICEF en este mismo informe, plantea la importancia de la educación en el desarrollo intelectual, emocional y social de los niños, las niñas y los adolescentes, el cual podría verse afectado con la incorporación al trabajo.

Agrega que este tiende a perjudicar el proceso de aprendizaje de esta población, en las siguientes formas:

- absorbe frecuentemente tanto tiempo que imposibilita la asistencia a la escuela:

- deja a los niños tan exhaustos que éstos carecen de la energía necesaria para asistir a la escuela o no pueden estudiar con suficiente rendimiento cuando están en clase;

- algunas ocupaciones, especialmente las labores agrícolas estacionales, causan pérdida de numerosos días de clase incluso de los niños que están escolarizados:

- el ambiente social del trabajo contribuye a socavar a veces el valor que conceden los niños a la educación, algo a lo que son especialmente susceptibles los niños de la calle;

- los niños maltratados en el lugar de trabajo quedan a veces tan traumatizados que no pueden concentrarse en las tareas escolares o son rechazados por los maestros como elementos perturbadores de la clase (p.25).

En este sentido la OIT apunta que:

El trabajo infantil es un obstáculo tanto para el desarrollo infantil como para oportunidades futuras. Todos los niños, sin excepción de raza o condición social y económica, tienen derecho a gozar sus años de infancia y desarrollarse plenamente y naturalmente. Todos tienen el derecho al amor, la educación y la protección. Conocer y entender estos derechos es el primer paso para prevenir el trabajo infantil y ofrecer a los niños una educación para que su futuro sea mejor (p.20).

\section{Situación del trabajo infantil en Costa Rica}

Un estudio realizado por la Municipalidad de San José (1999) refleja que, el cantón central de esta provincia es una zona de atracción para el trabajo informal de la población infanto-juvenil, que hay un predominio de menores de edad que trabajan para grupos familiares, principalmente compuestos por figuras parentales y que la mayoría de los niños y adolescentes que laboran en espacios públicos son menores de 15 años y presentan una alta exclusión del sistema educativo y extraedad académica. 
En este mismo sentido en el 2002, investigadores de la Universidad Nacional realizaron un estudio para determinar la cantidad de personas menores de edad económicamente activa, encontrándose que "del total de la fuerza de trabajo infantil y adolescente. un $5 \%$ es menor de 12 años, el $25 \%$ tiene entre 12 y 14 años y el 70\% son adolescentes entre 15 y 17 años de edad" (p.29). A criterio de los investigadores la inserción al mercado laboral es creciente con la edad.

De igual manera, la Fundación Paniamor (2002), hizo un estudio con el fín de conocer el número de niños, niñas y adolescentes que se dedican al trabajo infantil doméstico en hogares de terceros, "considerada una de las formas de explotación más generalizada, pero a la vez menos conocida y atendida" (p.9). Esta investigación permitió conocer que, del total de menores que participaron (250), la mayoría son mujeres, se insertan en el ámbito laboral entre los 13 y 15 años debido a las necesidades económicas en sus familias, las principales actividades que desempeñan son el aseo del hogar y el cuidado de otras personas menores de edad, "actividad expresamente prohibida" en el Código de la Niñez y la Adolescencia y entre otros datos significativos encontrados, cabe mencionar que específicamente en lo concerniente a la educación se tiene que:

Los indicadores referidos a su participación en el sistema educativo (niveles de escolaridad, repitencia, deserciọn y exclusión), cuestionan el cumplimiento de los principios de obligatoriedad, universalidad y gratuidad de la educación (...), (p.129).

Lo antes mencionado, es muy importante de analizar, para determinar si se está o no respetando el derecho de las personas menores de edad a tener un óptimo desarrollo integral, especialmente, si tomamos en cuenta los datos del Ministerio de Educación Pública (2003) en relación con el número de niños, niñas y adolescentes que desertaron del sistema educativo en el año 2002.

Al respecto se tiene que, en este mismo año hubo una deserción intra-anual en I y II ciclos, por dependencia pública, privada y privada subvencionada, de 21100 estudiantes (11711 niños y 9389 niñas). Además, segregando los datos por dependencia, se tiene que:

- La deserción intra-anual en I y II ciclos de los centros educativos públicos fue de 20716 estudiantes, de los cuales 11516 son niños y 9200 son niñas.

- La deserción intra-anual en I y II ciclos del sector educativo privado fue de 339 estudiantes (166 niños y 173 mujeres).

- La deserción intra-anual en I y II ciclos de la educación privada subvencionada fue de 45 estudiantes ( 29 niños y 16 niñas). 
- Así mismo, se encontró que la deserción intra-anual en III ciclo y Educación Diversificada, tanto Diurna como Nocturna y de los centros educativos públicos, privados y privados subvencionados, fue de 33014 estudiantes, de estos 18510 son hombres y 14504 son mujeres.

Con base en la información precitada cabe preguntarse ¿qué hacen y dónde están estos niños, niñas y adolescentes que desertan del sistema educativo costarricense? ¿Será la mayoría de estos los que engrosan el porcentaje de las personas menores de edad económicamente activas?

Sería interesante poder plantear y ejecutar una investigación a nivel macro. que permita conocer qué está ocurriendo con nuestro sistema educativo, el cual pareciera no está reteniendo a los estudiantes -al menos en el sector público-. Cabe pensar que, no se les están brindando alternativas innovadoras que satisfagan sus necesidades, intereses y expectativas consecuentes con la realidad en que están inmersos, lo que a su vez limita el uso de los conocimientos adquiridos en beneficio de su propio desarrollo integral y eventual desempeño laboral.

No obstante, como bien señala UNICEF (s.f.), esta no es una tarea exclusiva del sistema educativo; es una responsabilidad que nos compete a todos y todas, el velar para que los derechos de nuestros niños, niñas y adolescentes se cumplan.

\section{Referencias}

Carrillo, M. de los A. y Bomilla, V. H. (2002). Cartago: Dimensión, naturaleza y entorno socioeconómico del trabajo infantil y adolescente. Costa Rica: Programa Internacional para la Erradicación del Trabajo Infantil; Organización Internacional del Trabajo.

Dobles T., C. y Antezana R., P. (2002). El trabajo infantil y adolescente doméstico en Costa Rica. Costa Rica: Oficina Internacional del trabajo; Programa Internacional para la Erradicación del Trabajo Infantil.

Fondo de las Naciones Unidas para la Infancia. (1997). Educación y trabajo infanto-juvenil. Informe. Costa Rica: UNICEF.

Fondo de las Naciones Unidas para la Infancia. (1997). Estado Mundial de la Infancia 1997. Estados Unidos de América: UNICEF. 
Ministerio de Educación Pública. (2003). Estadísticas. Costa Rica: Departamento de Estadística.

Municipalidad de San José. (1999). Perfil del trabajo infanto jurenil en espacios públicos del Cantón Central de San José. Costa Rica: UNICEF.

Naciones Unidas. (1989). Convención sobre los Derechos del Niño. Asamblea General de las Naciones Unidas.

Organización Internacional del Trabajo. (1996). El trabajo infantil: ¿Qué hacer? Ginebra: Oficina Internacional del trabajo; Programa Internacional para la Erradicación del Trabajo Infantil.

Organización Internacional del Trabajo. (s.f.). Trabajo infantil, Derechos de los niños y Educación. Libro 1. Oficina Internacional del trabajo; Programa Internacional para la Erradicación del Trabajo Infantil.

Patronato Nacional de la Infancia. (1999). Principales disposiciones lega-

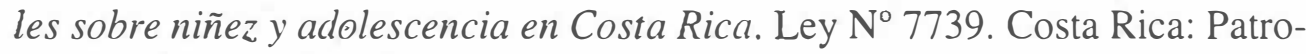
nato Nacional de la Infancia.

Trejos Z., Y. (1998). Programa para favorecer el desarrollo personal de los niños y niñas con necesidades educativas especiales, mediante el fortalecimiento de su autoestima. Costa Rica: Universidad Nacional. 